\title{
L'Accentuation dans le vers de Shakespeare
}

\section{Henri Suhamy}

Christophe Hausermann (éd.)

\section{(2) OpenEdition \\ Journals}

Édition électronique

URL : http://journals.openedition.org/shakespeare/2835

DOI : $10.4000 /$ shakespeare.2835

ISSN : 2271-6424

Éditeur

Société Française Shakespeare

Édition imprimée

Date de publication : 1 mai 2014

Pagination : 165-178

Référence électronique Henri Suhamy, « L'ACCENTUATION DANS LE Vers De SHAKESPEARE 》, Actes des congrès de la Société française Shakespeare [En ligne], 31 | 2014, mis en ligne le 01 mai 2014, consulté le 19 avril 2019. URL : http://journals.openedition.org/shakespeare/2835 ; DOI : 10.4000/shakespeare.2835 


\title{
L'ACCENTUATion Dans LE VERS DE SHAKESPEARE
}

\author{
Henri SUHAMY
}

\begin{abstract}
L'accentuation, en anglais comme dans les autres langues, est une donnée lexicale que les poètes exploitent en tant que moteur du rythme (notion à ne pas confondre avec le mètre, malgré la désastreuse confusion qui règne depuis plus de quatre-vingts ans), mais n'ont en principe pas la possibilité de modifier. La question est cependant plus complexe que cette simple constatation. II faut d'abord rappeler que la place de l'accent dans certains mots a changé depuis le $x v^{e}$ siècle, mais ce n'est là qu'un phénomène marginal. II faut surtout souligner que l'accentuation peut se plier aux schémas préétablis qui instaurent par exemple les rythmes iambique ou trochaïque. Le vers anglais utilise le rebond rythmique qui se produit dans les polysyllabes, ainsi que l'intonation qui parfois rehausse les monosyllabes. Shakespeare se montre particulièrement habile dans sa façon de permettre aux accents secondaires de trouver leur place dans la séquence rythmique, ou de procurer une rime, en les plaçant entre deux syllabes indiscutablement faibles, ou en bout de vers. Cela implique qu'un texte shakespearien doit être étudié comme une partition musicale avant d'être mis en voix, ce qui entraîne aussi que le rythme poétique n'a pas été conçu pour donner lieu à des analyses artificielles, segmentant les vers en séquences décousues de cellules appelées pieds, mais pour animer par sa pulsation interne les phrases que prononcent les acteurs sur la scène. Leur tâche est difficile, car ils doivent fondre dans un même creuset les linéaments fondamentaux de la langue parlée, l'expression des sentiments et l'architecture musicale des vers.
\end{abstract}

Accentuation in English as in other languages is a lexical feature that poets make use of in order to propel the rhythm (a notion which is not synonymous with metre, contrary to the disastrous confusion that has been prevailing for some eighty years or so by now), but that in theory they have no right to reshape at will. Yet the question is more complex than appears from this statement. First of all some words were stressed differently from nowadays in the sixteenth century, but this is only a side-issue. More important is the fact that admittedly accentuation can adapt itself to preconceived schemes, such as those that set up an iambic or trochaic rhythm. English verse capitalizes the rhythmical rebound produced in long words, as well as the intonation which sometimes heightens monosyllables. Shakespeare proves particularly clever in his handling of secondary stresses, when they are needed to uphold the regular rhythm, or to provide a rhyme, placing them deftly between definitely weak syllables, or at the end of a line. This implies that a Shakespearian text must be studied like a musical score before being voiced, and also that poetical rhythm was not invented in order to bring about artificial analyses that cut up lines into disconnected cells called feet, but to enliven, thanks to the pulsation running through the lines, the sentences delivered by the players on the stage. Actors have a difficult task, because they must fuse as in a crucible the fundamental data of the spoken language, the expression of feelings, and the musical architecture of verse.

$\mathrm{C}$

omme dans la plupart des langues l'accentuation constitue en anglais une donnée lexicale, le français constituant une exception. À première vue elle concerne les locuteurs s'exprimant dans cet idiome, et ne ressortit pas à des études de nature stylistique. Mais l'accentuation constitue aussi le principal moteur du rythme dans la poésie anglaise, et même en prose. Selon une définition élémentaire le rythme résulte de l'arrangement selon lequel se suivent les syllabes, plus ou moins voulu, ou instinctif, ou machinal. Les accents constituent donc la matière première du rythme, existant avant la mise en forme, de la même façon que les sons existent avant la musique. En principe l'auteur 
n'a ni le droit ni les moyens de façonner le langage comme de la pâte à modeler, ni de transformer à son gré la nature des accents ou la place qu'ils occupent dans les mots. Cela paraît aller de soi et pourtant il convient de ne pas s'enfermer dans des a priori contraignants. À l'inverse de ce qu'affirment certains phonéticiens selon lesquels le rythme poétique découle de la langue parlée, Jakobson a dit que le propre des systèmes de versification est de faire violence au langage. Il est permis à ce propos de critiquer les doctrines d'Enid Hamer, ${ }^{1}$ qui a commis deux contresens qui se sont incrustés dans les esprits, tout en prenant le relais de certaines doctrines contestables élaborées au XIx siècle. Premièrement l'axiome selon lequel les poètes n'ont jamais eu le droit ni la possibilité ni l'intention de modifier ou d'infléchir en quoi que ce soit l'accentuation lexicale. Deuxièmement la confusion entre le mètre et le rythme, alors que ces deux notions et les réalités qu'elles recouvrent, tout en travaillant en étroite coordination, comme les abscisses et les ordonnées dans un graphique, ne sont pas synonymes. Le mètre se réfère à la longueur ou durée des vers, le rythme à l'effet produit par les accents. La distinction et la coordination entre ces deux données se manifestent par exemple dans le fait que les mètres pairs, celui du décasyllabe par exemple, tendent à favoriser le rythme qu'on appelle iambique, comme dans le décasyllabe suivant ${ }^{2}$ :

The clock hath strucken twelve upon the bell

(The Comedy of Errors, I.ii.45)

tandis que les mètres impairs favorisent le rythme trochaïque, comme dans ces heptasyllabes récités par Puck :

Now the hungry lion roars

And the wolf behowls the moon;

Whilst the heavy ploughman snores,

All with weary task fordone.

Now the wasted brands do glow,

\footnotetext{
1 Enid Hamer est l'auteur de The Metres of English Poetry, Londres, Methuen, 1930. La confusion entre le mètre et le rythme - plus exactement l'utilisation du mot metre à la place de rhythm - est annoncée par le titre et se poursuit dans tout l'ouvrage. L'auteur a fait école et à peu près tous les spécialistes ou supposés tels la commettent, avec pour conséquence que la réalité du mètre au sens propre (qui concerne la durée ou la longueur des vers, comme unités du discours versifié) se trouve escamotée. Cette confusion déplorable a atteint la France et même la musicologie, comme on peut le constater à la lecture d'un livre récent de Gilles Cantagrel, Les Cantates de Bach (Paris, Fayard, 2010) où le mot mètre remplace à chaque fois le mot rythme.

${ }^{2}$ Les références chiffrées sont tirées des éditions Arden.
} 


\author{
Whilst the screech-owl, screeching loud, \\ Puts the wretch that lies in woe \\ In remembrance of a shroud. \\ (A Midsummer Night's Dream, v.i.368-75)
}

J'ai accentué des monosyllabes grammaticaux comme la conjonction and et les prépositions in et of. S'agit-il d'une prononciation artificielle ? Oui et non. En prose ou dans la conversation, les accents ajoutés paraîtraient incongrus, mais le propre de la poésie versifiée est de faire apparaître comme naturel ce qui sonnerait artificiel dans un autre contexte. Le rythme crée une mécanique répétitive. Mû par les accents, une fois mis en marche, le mouvement continue; dans sa marche forcée, il entraîne des syllabes qui en temps normal resteraient phonétiquement discrètes. Il est vrai que l'effet d'entraînement se fait sentir plus aisément dans les séquences trochaïques que dans les séquences iambiques, au point que même en français on se laisse aller à suivre le mouvement, comme dans certaines comptines. Le rythme trochaïque est désigné comme descendant, c'est une cadence au sens étymologique, contrairement au rythme iambique, ascendant, allant de la faible à la forte, ce qui demande un effort, une obéissance à des normes imposées au discours, car il va à contre-courant du langage ordinaire. Sur la question des normes, il faut se garder de passer d'un extrémisme à l'autre, dans un formalisme qui au nom de l'idéal poétique, se donnerait le droit de créer un langage artificiel. La poétique a peut-être le pouvoir de transformer la langue commune en quelque chose de riche et d'étrange, non de la métamorphoser de façon à la rendre méconnaissable. L'accentuation en anglais s'inscrit dans une sorte de fourchette, étroite mais permettant une certaine souplesse, une adaptabilité que les poètes savent exploiter.

Avant de continuer sur ce leitmotiv, il convient de poser deux questions générales, et d'essayer d'y répondre, brièvement. La première concerne la nature de l'accentuation. Qu'est-ce qu'un accent tonique ? Faut-il prendre littéralement la dénotation contenue dans le mot tonique, qui se réfère à l'échelle des sons plutôt qu'à des différences dynamiques, en termes acoustiques, à la fréquence plus qu'à l'amplitude ? Georges Faure, phonéticien savant mais théoricien aventureux, a soutenu qu'en anglais comme dans certaines autres langues l'accent se traduit par une élévation de la voix, non en intensité mais en tessiture. Autrement dit les syllabes accentuées se situent dans les zones aiguës et les syllabes inaccentuées dans les zones graves. Il en 
a déduit que le mouvement de la voix dans la récitation poétique s'apparentait à une mélodie plutôt qu'à un battement alternatif. Sa thèse ne s'est pas imposée, il a commis des erreurs, a manqué d'information historique, et a poussé trop loin certaines de ses théories, mais il faut le remercier d'avoir écrit qu'un texte poétique n'a pas à ressembler à un message en morse, où alternent des signaux fixes et contrastés, et d'avoir souligné l'importance des accents intonatifs. Il faut admettre cependant que même si le rythme poétique n'est pas battu avec une monotonie percussive, il reste perceptible, sinon on ne pourrait pas en parler.

L'autre question préalable concerne la diachronie, et la langue de Shakespeare, avant d'en arriver au langage poétique et théâtral. L'anglais se prononçait-il en son temps et dans toutes les régions d'Angleterre de la même façon qu'aujourd'hui ? Certainement pas, mais si les phonèmes ont évolué, il n'en va peut-être pas de même pour l'accentuation. Elle représente sans doute l'élément le plus stable de la langue parlée, aussi bien dans le temps que dans l'espace. Il y a des exceptions, même dans la synchronie, par exemple le mot controversy donne lieu à une controverse, car certaines personnes prononcent controversy et d'autres controversy. Dans Shakespeare c'est controversy qu'on trouve, et nous le savons grâce notamment au vers de Cassius :

And stemming it with hearts of controversy;

(Julius Caesar, ..ii.108)

avec en plus la quasi-certitude que le mot contient deux accents au lieu d'un : controversy, prononciation qui n'est pas incompatible avec ce qu'on entend dans la conversation ordinaire. Comment connaissonsnous la façon dont Shakespeare lui-même prononçait ses vers ? Il n'a pas laissé d'enregistrements, mais si l'accentuation constitue le moteur du rythme, celui-ci obéit avec plus ou moins de docilité à des grilles préétablies, en l'occurrence la grille iambique, qui fait tomber les accents sur les syllabes paires. Elle n'est pas toujours suivie de façon mécanique, mais on sent bien que ce vers sonnerait de façon boiteuse si l'on accentuait le mot controversy autrement. L'accentuation des mots n'est pas restée dans tous les cas inchangée au cours des siècles, il y a des exceptions au pacte de stabilité qu'elle semble avoir respecté le plus souvent. Dans son ouvrage monumental sur la prononciation de Shakespeare le philologue américain Helge Kökeritz a recensé environ 
300 mots dont l'accentuation diffère de celle d'aujourd'hui. Cela ne représente pas un grand pourcentage, même si ce pourcentage se calcule sur un total excluant les monosyllabes, nombreux en anglais. Par exemple le mot exile était accentué sur la deuxième syllabe, comme dans:

Now my co-mates and brothers in exile, (As You Like It, II.i.1)

On prononçait sans doute /ek'si:l/ mais c'est de l'accentuation qu'il est question ici, non des phonèmes, donc laissons ceux-ci de côté. Étant donné que les décasyllabes iambiques sont quasi obligatoirement accentués sur la dixième syllabe, on peut déduire, ou induire que dans le mot en question l'accent tombe sur la deuxième syllabe. L'œuvre de Shakespeare contient 32 occurrences du mot exile, verbe ou substantif, et toutes confirment cette accentuation. Parfois l'évolution s'est faite dans le sens inverse, certains mots sont accentués sur la première syllabe, contrairement à l'usage actuel, par exemple supreme, mot utilisé assez souvent, comme dans :

The life of purity, the supreme fair, (The Rape of Lucrece, 780)

Cela concerne aussi des mots de trois ou quatre syllabes, comme empirics dans All's Well That Ends Well (п.і.121) :

To empirics or to dissever so,

Ou confessor dans Measure for Measure (iv.iii.128)

One of our convent, and his confessor,

Le cas du mot obscure est intéressant. En tant que verbe, il est accentué sur la seconde syllabe, comme dans ce vers de Henry $V$ :

And so the prince obscured his contemplation

alors que dans sa fonction d'adjectif, c'est la première syllabe qui porte l'accent, comme dans ce vers de Hamlet :

His means of death, his obscure funeral

(Iv.vi.210)

Le vers qui vient d'être cité permet de passer à un autre chapitre, plus important que le précédent, car il a pour objet non la diachronie mais la technique et l'esthétique du vers. La question concerne non ce qui 
différencie l'anglais élisabéthain et l'anglais d'aujourd'hui, mais la langue versifiée de la langue courante. Il n'existe pas de consensus unanime sur ce point, mais l'opinion la plus répandue chez les spécialistes est que dans le vers cité précédemment, le mot funeral a deux accents et non un seul. À sa qualité de proparoxyton il ajoute celle d'oxyton. La syllabe finale de ce mot, sur laquelle pèse un accent secondaire, serait même peut-être allongée si le texte était mis en musique. Cet accent provient d'un rebond rythmique, mais dans le langage courant, et dans les diverses façons de prononcer ou de rythmer la langue anglaise, on ne l'entend pas toujours. Il ne résulte cependant pas d'un règlement arbitraire et académique. Il appartient au rythme naturel de la langue anglaise, même s'il reste souvent à l'état virtuel. Dans les vers de type traditionnel, notamment dans ceux de Shakespeare, il constitue un outil indispensable, au point qu'un mot de cinq syllabes, comme dans

But soft, here come my executioners

(Richard III, I.iv.339)

peut fournir trois accents, même si tous ne sont pas frappés avec la même intensité, ou sur la même note de la gamme vocale.

Si les polysyllabes peuvent donner lieu à un consensus assez large, la question des monosyllabes est plus délicate. Les théoriciens de la versification, ceux notamment qui enseignent la diction aux apprentis acteurs, doivent faire preuve à la fois de doigté et de fermeté. On trouve souvent des vers entièrement formés de monosyllabes. Il y a des cas où l'accentuation s'impose naturellement, par exemple dans ce vers de Lucrece (617) :

And wilt thou be the school where lust shall learn?

Les positions paires, normalement accentuées dans le schéma iambique, sont occupées la première par l'auxiliaire wilt, qui porte un accent intonatif, les suivantes par des substantifs, school et lust, et des verbes, be et learn, normalement accentués. Mais il existe d'autres cas, notamment dans les dernières œuvres, où la mécanique binaire s'assouplit et où les séquences de monosyllabes tendent à remplacer la marche rythmique par une ligne mélodique, non forcément suave, car elle peut être heurtée à la manière d'un récitatif, comme dans ce fragment d'un dialogue entre Leontes et Mamillius : 
LEON. Art thou my calf?

MAM. Yes, if you will, my lord.

LEON. Thou want'st a rough pash and the shoots that I have

To be full like me: yet they say we are

Almost as like as eggs.

(The Winter's Tale, r.ii.127-9)

Un vers parmi d'autres constitue un exemple des questions qui se posent, celui que prononce Henry V, dans la tirade d'Azincourt,

But we in it shall be remembered...

(Henry V, rv.iii.59)

Il contient des caractéristiques propres à illustrer les linéaments de l'accentuation prosodique. Si l'on applique de façon formelle la grille décasyllabique autant que le schème iambique on est amené à prononcer le participe passé remembered en quatre syllabes, et en plus à accentuer la désinence. La plupart des acteurs le font encore aujourd'hui, mais souvent en accentuant la syllabe finale du bout des lèvres, de façon un peu fantomatique, comme pour faire savoir qu'ils connaissent la tradition mais veulent éviter un académisme ostentatoire. Ce ne sont pas seulement les accents lexicaux ou ceux qu'exige le schéma rythmique qui alimentent le rythme, mais les accents intonatifs. Le pronom we a manifestement une fonction emphatique dans le discours, car ce we flatteusement collectif, abolissant la distance qui sépare le monarque de ses sujets, est rehaussé par l'intonation. Il trouve ainsi sa place dans la séquence rythmique. Il est aidé en cela par la syntaxe, car non suivi du verbe, il est affranchi de la position proclitique qui est habituellement celle des pronoms sujets et les prive de toute possibilité d'accentuation ; il en va de même du pronom it, accentué pour une fois, car libéré de son habituelle position proclitique ou enclitique, il est complément indirect et non sujet. Dans sa fonction anaphorique, il remplace le this day du vers précédent. Cela lui donne assez de poids pour porter un accent, nécessaire sur la quatrième syllabe, qui constitue toujours l'un des deux piliers rythmiques du vers dans Shakespeare, l'autre étant la dixième. À propos de dixième syllabe, la désinence du participe passé remembered rappelle un détail qui peut troubler les usagers. Quatre vers plus haut dans la même tirade on entend ceci :

Be in their flowing cups freshly remember'd. (Henry $V$, Iv.iii.55)

C'est un vers de 11 syllabes, la onzième étant inaccentuée, donc formant une terminaison féminine. Dans le vers cité précédemment, comme 
toujours le mètre et le rythme travaillent en étroite collaboration, ce qui a pour conséquence que non seulement la désinence finale est articulée mais qu'en plus elle est accentuée. Avec quelle intensité ? Accordons à l'acteur une certaine latitude. Cela dit, les variantes phonétiques embarrassent parfois les interprètes, comme dans le cas du fameux banished de Romeo and Juliet. Tantôt ce participe se prononce banish'd, tantôt banished, et le Romeo de service a souvent du mal à s'y retrouver. Rappelons aussi que la désinence prononcée n'est pas forcément accentuée, comme dans

But she perforce withholds the loved boy,

(A Midsummer Night's Dream, ir.i.26)

où il faut dans loved accentuer le radical du verbe, non la désinence. Cela paraît évident et pourtant on a pu lire, imprimés noir sur blanc, des commentaires explicatifs où étaient confondues articulation et accentuation. 3

Il y a des cas où l'accentuation sur la désinence peut sembler étrange et artificielle, provoquant une réaction de refus. Dans ces deux vers prononcés par Juliet :

Go ask his name. If he be married My grave is like to be my marriage-bed.

(Romeo and Juliet, I.v.133-4)

Le participe married est allongé par diérèse en marr-i-ed pour satisfaire simultanément le mètre (allonger le vers jusqu'à dix syllabes), le rythme (placer un accent sur la dixième syllabe, en vertu du principe de rebond accentuel dans les mots polysyllabiques), et la rime avec bed, pour respecter la règle, intangible dans Shakespeare, selon laquelle seules les syllabes accentuées riment entre elles. Lovéd ne peut pas rimer avec bed, mais married le peut. Cela dit, faire rimer married et bed peut sembler difficile à certaines actrices et à certains metteurs en scène, y compris chez ceux qui insistent sur la théâtralité, c'est-à-dire l'artificialité du spectacle, craignant peut-être que cette bizarrerie ostensiblement passéiste détourne l'attention du public de ce qui se

3 Erreur commise par Paul Bacquet dans l'introduction à Gorboduc de Sackville et Norton (édition bilingue, Paris, Aubier Montaigne, 1976), page 58. Il donne entre autres exemples le vers suivant « That loved him more dearly than herself ? » (IV.1.80) pour affirmer que dans loved c'est la désinence ed qui doit être accentuée. Articulée, oui, mais accentuée, c'est impossible phonétiquement et ce serait contradictoire avec le rythme iambique du vers. 
passe sur la scène. Qu'en était-il à la fin du xvi ${ }^{\mathrm{e}}$ siècle ? Il est difficile de le savoir avec précision. Dans le langage courant on prononçait certainement married comme aujourd'hui, mais sans doute la diérèse était-elle plus facilement qu'aujourd'hui acceptée par le public. Dans le fameux vers qui sert de leitmotiv à Mark Antony :

But Brutus says he was ambitious

(Julius Caesar, iII.ii.88)

la diérèse qui donne quatre syllabes et deux accents au mot am-bi-ti-ous et que malheureusement les acteurs ne respectent presque jamais, ne se justifie pas seulement pour des raisons de métrique formelle. Elle accentue l'ironie hargneuse du discours. On sent quelque chose de méprisant dans cet écho étiré du mot prononcé par Brutus, dans le chuintement huileux de la chute du vers, ambiti-ous. Les deux visages différents, voire contrastés de la rhétorique apparaissent en même temps : stylisation esthétique du discours, et intention persuasive, conative, voire performative. Notons au passage l'accent à la fois rythmique et emphatique sur le verbe was. Il faut noter aussi que les diérèses dans Shakespeare ne sont jamais prévisibles. Les mêmes mots peuvent se prononcer avec ou sans diérèse. Le mot ambition n'a généralement que trois syllabes et un seul accent, mais il s'allonge parfois et gagne un accent comme dans ce vers de King John:

Are capable of this ambition...

(II.i.476)

Signalons au passage que la syllabe finale du mot capable fournit un accent au vers, ce qui nous met sur la voie de l'épenthèse. L'épenthèse, ou anaptyxis, consiste à ajouter une voyelle non écrite entre deux consonnes, une sorte de tampon amortisseur, comme en français quand on prononce le vingt-e-deux mars-e de cette année, pour lubrifier l'articulation. Dans le sonnet 106, comment le participe passé disabled peut-il rimer avec un autre participe passé sans doute inventé par Shakespeare, à savoir strumpeted ? Il faut dire dis-a-bel-ed. Ces diérèses et épenthèses, qu'on peut qualifier de chevilles, accompagnées d'une accentuation volontariste, deviennent moins nombreuses à mesure qu'on avance dans la carrière de Shakespeare. Est-ce dû à une évolution de la langue anglaise au cours de sa vie, ou à une mutation progressive de son style? Il est difficile de le savoir, mais je penche pour la deuxième hypothèse. 
Le principe de l'alternance accentuelle produisant un rebond rythmique a parfois des conséquences étranges, notamment sur les mots composés contenant trois syllabes. Par exemple dans le vers suivant, tiré de The Taming of the Shrew (v.iv.41) :

Allots thee for his lovely bedfellow.

D'après les critères métriques et rythmiques c'est la syllabe finale de bedfellow qui doit porter un accent, alors qu'isolé le mot fellow est accentué sur la première. Ce détail fut remarqué par un universitaire du nom de Goswin König, qui publia à Strasbourg, en 1888, un ouvrage intitulé Der vers in Shakesperes Dramen. ${ }^{4}$ J'ai relevé 56 occurrences de ce phénomène, qui n'apparaît que dans les œuvres destinées au théâtre, et qui portent sur des mots composés comme torch-bearer, bloodsuckers, archbishop, grandfather, ring-leader, sheep-shearing ${ }^{5}$. Cette particularité n'est pas inconnue en anglais moderne, si l'on considère que a Frenchwoman en un seul mot ne se prononce pas tout à fait de la même façon que a French woman en deux mots, mais on ne va pas jusqu'au rebond rythmique observé plus haut, et qu'on trouve appliqué à Marguerite d'Anjou dans 2 Henry VI (..iii.140). On peut juger cette accentuation virtuelle ou facultative plutôt qu'obligatoire. On peut excuser ou même approuver les acteurs du XxI ${ }^{\mathrm{e}}$ siècle s'ils n'infléchissent pas toujours la prononciation des vers dans le sens d'une reconstitution historique qui peut se heurter à des objections ; il n'est pas impossible que Shakespeare ait pratiqué des expédients pour sauver les apparences. On peut dans certains cas invoquer l'opinion du poète lauréat Robert Bridges qui a écrit dans son livre sur la versification de Milton qu'en matière d'élocution la pratique et la théorie divergent parfois. ${ }^{6}$

\footnotetext{
4 Goswin König, Der Vers in Shakesperes Dramen, Strasbourg, 1888, p. 66.

5 Voici, en note la liste complète, par ordre de fréquence : bed-fellow, torch-bearer, grandfather, school-master, play-fellow, gunpowder, house-keeping, bed-chamber, bloodsuckers, cup-bearer, godfather, archbishop, bag-piper, bed-swerver, bilberry, bloodshedding, cave-keeper, coach-makers, codpieces, crow-keeper, eaves-dropper, forthcoming, Frenchwoman, grandmother, grasshopper, madwoman, midsummer, newsmongers, primroses, sheep-shearing, stepmothers. Les mots ci-dessus sont tous situés en fin de vers, mais trois autres, placés à l'intérieur d'un vers, ressortissent au même type d'accentuation : law-breaker, peace-makers, ring-leader.

6 Robert Bridges (1844-1930), devenu poète-lauréat en 1913, publia en 1889 Milton's Prosody, with a Chapter on Accentual Verse and Notes, OUP. La phrase où il dit (p. 18) que les grilles rythmiques et métriques du vers avaient chez Milton un caractère théorique et ne correspondaient pas forcément à ce qu'on exprime à haute voix, "Milton came to scan his verse one way and read them another » est citée, accompagnée d'un commentaire sarcastique, par Georges Faure dans son livre intitulé Les éléments du rythme poétique en
} 
Un détail caractéristique de l'habileté de Shakespeare en tant qu'artisan du vers apparaît dans sa façon de gérer les accents secondaires. Il y a dans ceux-ci une nuance de fragilité et de relativité qui risque de les placer en porte-à-faux. Tout d'abord, l'accent secondaire est toujours placé en position de force, c'est-à-dire, dans les vers iambiques, sur les syllabes paires, 2, 4, 6, 8, 10. C'est aux accents principaux, éventuellement intonatifs, que revient la charge de créer des variations rythmiques, notamment des alourdissements spondaïques, fréquents dans les vers constitués de monosyllabes, comme dans ce passage de Richard II :

I live with bread like you, feel want, Taste grief, need friends.

(III.ii.175-6)

Ensuite Shakespeare s'arrange pour qu'un accent secondaire, chargé de soutenir le rythme, ne risque pas de se heurter à plus fort que lui. Le procédé le plus simple consiste à placer un accent secondaire en fin de vers, comme celui du mot funeral cité précédemment ; la syllabe finale suit un phonème inaccentué, et devant elle se trouve un vide ; elle ne risque donc pas le sort inconfortable du pot de terre côtoyant le pot de fer. Dans ce vers :

To wall thee from the liberty of flight; $\quad$ (1 Henry VI, rv.ii.24)

la syllabe finale du mot liberty doit être accentuée, légèrement mais perceptiblement ; comme elle est placée entre deux syllabes inaccentuées, le coup de pouce accentuel permet de soutenir le rythme binaire sans créer un déséquilibre phonétique. La règle vaut aussi pour les monosyllabes. Dans le même vers la préposition from est redevable d'un accent, et l'auteur a pris soin de lui aménager un environnement propice, contrairement à ce qui se passe pour of, placé en neuvième position, et adossé à une syllabe manifestement accentuée. À l'exception des articles, les monosyllabes grammaticaux peuvent donc, selon les besoins, assumer un accent ou rester discrets. Dans

From fairest creatures we desire increase

(Sonnet 1)

le pronom we porte un accent, qui n'a pas la fonction emphatique qu'il a dans un autre exemple cité précédemment (But we in it shall be

anglais moderne : Esquisse d'une nouvelle analyse et Essai d'application au «Prometheus Unbound » de P.B. Shelley, La Haye et Paris, Mouton, 1970, p. 21. 
remembered). Il ne faut pas trop peser sur lui, mais faire sentir sans ostentation qu'il joue son rôle dans la séquence iambique. Un texte de Shakespeare doit être lu et déchiffré attentivement, comme une partition musicale, avant l'exécution.

Au cours des tentatives en vue de définir des notions simples et pourtant fuyantes comme le rythme, l'accentuation, l'intonation, un mot n'a pas été prononcé, celui de scansion. Il a son importance, il convient de ne pas l'esquiver. C.S. Lewis disait que l'essentiel dans le domaine de la versification est de savoir comment se scande un vers. ${ }^{7}$ Mais que signifie scander un vers ? Cela consiste-t-il à lui imposer une grille préconçue, même si le tissu phonétique se montre récalcitrant ? Ou bien, selon la méthode popularisée par Enid Hamer, à la suite de George Saintsbury ${ }^{8}$, à segmenter chaque vers en tronçons appelés pieds, et à donner à chacun d'eux un nom emprunté à la terminologie gréco-latine : iambes, trochées, pyrrhiques, spondées, anapestes, dactyles, amphibraques, tribraques, molosses, bacchius, antibacchius ? Rien que dans l'œuvre de Shakespeare, en s'en tenant au canon traditionnel, on trouve plus de 80 ooo vers, exactement 81600 , ce qui représente un potentiel d'environ 400 ooo pieds à étiqueter minutieusement, de quoi occuper pendant plusieurs années les podologues, podolâtres et podocrates. Il ressort des analyses en pieds, dans lesquelles se complaît une certaine tradition universitaire, des sortes de radiographies qui font apparaître comme des squelettes de dinosaures où se juxtaposent des vertèbres de tailles différentes et de formes hétéroclites. Seulement au lieu d'être fondées sur des appareils scientifiques et objectifs, les radiographies en question reflètent le plus souvent ce que le praticien a prévu d'y trouver, sans se préoccuper de l'état de la langue dans laquelle s'exprimait l'auteur, et des habitudes qui avaient cours en son temps ou qui lui étaient particulières. Une autre méthode consiste à travailler dans la continuité plutôt que dans la segmentation, car le staccato rythmique ne doit pas empêcher la fluidité du discours. Dans la bouche des grands acteurs les accents ne constituent pas un halètement de pulsations en relief, mais plutôt les points d'appui soutenant la déclamation

7 C.S. Lewis, « Metre », The Review of English Literature, ${ }^{\circ}{ }^{\circ} 1,1960$, p. 45.

8 Enid Hamer et George Saintsbury diffèrent cependant sur un point essentiel, à savoir que ce dernier admettait l'existence de grilles préconçues, tandis qu'Enid Hamer pensait qu'il faut d'abord prononcer les mots comme dans le langage ordinaire, puis découvrir ensuite le schéma qui résulte de cette lecture spontanée. 
dramatique autant que poétique. De plus ces accents s'intègrent au grain de la voix, à l’intonation personnelle des interprètes, qui conservent leur individualité reconnaissable même quand ils se moulent à la fois dans un système de versification imposé et dans le caractère du personnage auquel ils doivent s'identifier. Plutôt qu'étudier le rythme sur le papier, le faire à voix haute, comme les acteurs, à qui est destinée la poésie dramatique, et qui pour éviter aussi bien le message en morse que le récitatif aléatoire, doivent trouver à chaque instant un compromis, ou mieux, une synthèse, entre des données parfois divergentes : les linéaments fondamentaux de la langue parlée, l'expression des sentiments, et l'architecture musicale du vers.

\section{BIBLIOGRAPHIE}

Dobson, E.J., English Pronunciation 1500-1700, 2 vols, Oxford, OUP, 1957.

Faure, Georges, Recherche sur les caractères et le rôle des éléments musicaux dans la prononciation anglaise. (Essai de description phonologique), Paris, Didier, 1962.

-- Les Éléments du rythme poétique en anglais moderne, Paris, Mouton, 1970.

Jakobson, Roman, Éléments de poétique, Paris, Éditions de Minuit, 1957.

Kingdon, Roger, The Groundwork of English Intonation, Londres, Longmans, 1958.

Kökeritz, Helge, Shakespeare's Pronunciation, Newhaven, Yale University Press, 1953.

König, Goswin, Der Vers in Shakesperes Dramen, Strasbourg, K.J. Trübner, 1888.

Le Page, R.B., “The Dramatic Delivery of Shakespeare's Verse”, English Studies, ${ }^{\circ}$ 32, 1951.

Lewis, C.S., "Metre", The Review of English Literature, ${ }^{\circ}{ }^{1,} 1960$.

Saintsbury, George, A History of English Prosody from the Twelfth Century to the Present Day, Londres, Macmillan, 3 vols., 19061910.

Sipe, Dorothy L., Shakespeare's Metrics, Newhaven, Yale University Press, 1968. 
178 HENRI SUHAMY

Suhamy, Henri, Le vers de Shakespeare, Paris, Didier-érudition, 1983. --Versification anglaise, Paris, Ellipses, 1999.

Henri SUHAMY Université Paris Ouest Nanterre La Défense 